\title{
Ocular and Systemic Safety Evaluation of Calcium Formate as a Dietary Supplement
}

\author{
Michael M. Altaweel, ${ }^{1}$ Robert P. Hanzlik, ${ }^{2}$ James N. Ver Hoeve, ${ }^{1}$ Janis Eells, ${ }^{3}$ and Baoyan Zhang ${ }^{1}$
}

\begin{abstract}
Purpose: The objective of this study was to perform a preliminary evaluation of the ocular and systemic safety of calcium formate, a dietary calcium supplement for prevention and management of osteoporosis. Although formate is an endogenous product of metabolism, high concentrations are associated with toxicity during methanol overdose.

Methods: In this prospective clinical trial, 12 healthy women ingested calcium formate $(1,300 \mathrm{mg})$ three times a day for 14 days. Study evaluations included physical and ocular examination, extensive laboratory testing, serum calcium and formate levels, Early Treatment Diabetic Retinopathy Study (ETDRS) visual acuity, color vision, visual fields, visual evoked potential (VEP), and full-field, pattern, and multifocal electroretinograms (MERG).

Results: The mean baseline serum level of formate was $0.572 \pm 0.06 \mathrm{mM}$. Peak serum levels and final serum formate did not differ significantly from baseline. The final concentration was $0.582 \pm 0.091 \mathrm{mM}$. Accumulation of serum formate did not occur. There was also no evidence of toxicity with calcium formate ingestion. All examinations and tests remained normal, including optic nerve and retinal function. Three subjects had mild transient symptoms attributable to any calcium formulation.

Conclusions: Calcium formate is highly bioavailable and well-tolerated. Serum formate remained at basal levels and did not accumulate with repeated dosing. Systemic and ocular safety was demonstrated by objective testing. Given its high oral bioavailability, calcium formate may be a good choice for calcium supplementation in the prevention and management of osteoporosis. Further study will be needed to evaluate its long-term safety in a larger group of subjects representing more varied age, health, dietary, and nutritional status.
\end{abstract}

\section{Introduction}

C ALCIUM SUPPLEMENTATION IS USEFUL in the prevention of osteoporosis, a disorder of bone in which bone mineral density is reduced and bone microarchitecture is altered leading to bone fragility and a predisposition to fractures. ${ }^{1}$ The underlying mechanism in osteoporosis is an imbalance between bone resorption and bone formation, both of which are heavily influenced by nutritional and hormonal factors. ${ }^{2}$ Because of its hormonal component, menopausal (ie, estrogen deficient) women comprise the largest group of individuals suffering from osteoporosis. In the United States, $>10$ million people have osteoporosis and 34 million have osteopenia, or low bone mass, the precursor condition for osteoporosis. ${ }^{3}$ Osteoporosis is responsible for millions of fractures annually, primarily involving the lumbar vertebrae, hip, and wrist. The incidence increases with age and the 1-year mortality rate following hip fracture is $\sim 20 \%$.

Dietary calcium supplementation, which has been found to increase bone density and diminish the risk of associated fractures, is an important aspect of the prevention and management of osteoporosis. ${ }^{4-7}$ Calcium formate is a novel dietary calcium supplement ${ }^{8}$ with superior bioavailability compared with the most commonly used supplements such as calcium carbonate and calcium citrate. ${ }^{9}$ Although formate is a normal endogenous metabolite, sustained high concentrations of serum formate, such as those occur in cases of poisoning by methyl alcohol (wood alcohol), can be toxic, especially to the retina and optic nerve..$^{10-12}$ These effects

\footnotetext{
${ }^{1}$ Department of Ophthalmology and Visual Science, School of Medicine and Public Health, University of Wisconsin-Madison,
} Wisconsin.

${ }^{2}$ Department of Medicinal Chemistry, University of Kansas, Lawrence, Kansas.

${ }^{3}$ Department of Health Sciences, University of Wisconsin-Milwaukee, Wisconsin. 
manifest as reductions in electroretinogram and visual evoked potential (VEP) responses. ${ }^{13,14}$

In view of its potential use as a dietary supplement, the current study was designed to evaluate the safety of daily ingestion of calcium formate and to determine if serum formate accumulates with repeated dosing. The safety evaluation included sensitive objective testing of retinal and optic nerve function.

\section{Methods}

Twelve healthy adult females participated in this prospective open-label clinical trial (NCT00204893). The protocol adhered to the tenets of the Declaration of Helsinki and was approved by the University of Wisconsin Institutional Review Board. Signed written informed consent was obtained. A safety board was convened to review potential adverse events. Subjects were monitored throughout their participation for adverse events and adherence to protocol. A tablet count was performed upon completion of the study.

Inclusion criteria included: (1) age between 19 and 65; (2) body mass index between 18 and 27; (3) normal physical examination and normal electrocardiogram; (4) normal ocular examination and normal assessment of function; (5) normal clinical laboratory tests; (6) use of two forms of contraception, or postmenopausal status for 12 months prior to study entry, or surgical sterility, and negative serum pregnancy test. Exclusion criteria were: (1) prior history or current finding of significant ocular abnormality such as optic nerve disease, glaucoma, or retinal disease; (2) ocular surgery within 6 months of study entry; (3) abnormality in screening tests of visual function.

Subjects had baseline examination and testing followed by evaluations on days 1, 2, 3, 8, and 15. Evaluations included full physical examination, ECG, testing of respiratory function with peak flow meter and forced expiratory velocity, assessment of pupillary responses, dilated ocular examination, and fundus photographs. Blood samples were obtained at baseline and day 15 for $\mathrm{CBC}, \mathrm{PTH}$, thyroid status, total calcium, ionized calcium, formate, electrolytes, liver function, glucose, amylase, total protein, albumin, BUN, and creatinine. Serum calcium was tested on selected visits, prior to the first dose of the day. Tests of visual function included visual field, color vision test, full-field ERG, pattern ERG, multifocal ERG, flash VEP, and pattern-reversal VEP.

Each subject ingested 1,300 $\mathrm{mg}$ of calcium formate $\left[\mathrm{Ca}\left(\mathrm{HCO}_{2}\right)_{2}\right]$ three times a day for 14 days (total daily dose $=$ $3,900 \mathrm{mg}$ of calcium formate $=1,200 \mathrm{mg}$ of elemental calcium $+2,700 \mathrm{mg}$ of formate). Serum formate concentrations were obtained from venous blood samples. Centrifuged supernatant was deproteinized by adding $30 \mu \mathrm{L}$ of $7.5 \% \mathrm{ZnSO}_{4}$ and $30 \mu \mathrm{L}$ of $0.4 \mathrm{~N} \mathrm{NaOH}$ per $100 \mu \mathrm{L}$ of serum followed by further centrifugation. Formate concentrations were determined on this supernatant using the fluorometric assay of Makar and Tephly. ${ }^{15}$ To test for accumulation of serum formate, morning fasting blood samples were obtained at screening and prior to the first dose on study days 1, 2, 3, 8, and 15. Blood samples for peak serum formate were obtained 40-60 min after ingestion of the second dose on days 1,2,3, and 8 .

Visual acuity testing was performed with Early Treatment Diabetic Retinopathy Study (ETDRS) refraction and retroilluminated Ferris-Bailey charts. Stereoscopic fundus photographs were evaluated by masked graders for the presence of retinal edema, hemorrhage, and optic nerve abnormality. Color vision was assessed with the FM-100 Hue test. Visual field testing utilized the automated standardized Humphrey 24-2 using the Swedish Interactive Threshold Algorithm.

Electroretinographic (ERG) testing conformed to the standards set forth by the International Society for the Clinical Electrophysiology of Vision. Testing was performed with a UTAS-3000 (LKC Instruments, Gaithersberg, MD) visual electrodiagnostic device. ERG testing obtained intensity versus response functions under dark-adapted (scotopic) and light-adapted (photopic) conditions to test retinal function.

The pattern ERG (PERG) assessed ganglion cell function. Recording was performed with chessboard pattern stimuli that exchange black and white squares at a rate of $2 \mathrm{~Hz}$. The peak latency and amplitude of response waves were determined. VEPs were recorded from scalp electrodes to evaluate optic nerve function, measuring the latency and amplitude of the positive wave near $100 \mathrm{~ms}$. Baseline and follow-up tests were compared by time group and for individual subjects across time. The selection of sample size was based on the VEP test, with normal variation being $\pm 5 \mathrm{~ms}$, and a delay of $8 \mathrm{~ms}$ considered definitely abnormal. Having 12 subjects allowed a $90 \%$ power to detect a significant difference in this critical test with $P<0.05$.

Multifocal ERG (MERG) was performed to provide additional topographic information regarding retinal function. ${ }^{16,17}$ This test used the Visual Evoked Response Imaging System (version 4.9, EDI Inc., CA) with dilated pupils. Both eyes were tested simultaneously. The 103 first-order kernel MERGs were reduced to six waveforms that were the average of six concentric rings from the fovea. The peak latency and amplitude of the response waves were scored using an automated routine written in Matlab ${ }^{\mathrm{TM}}$ (Natick, MA). Averages of the six rings across the 12 subjects were compared across baseline, postdose day 3 and day 15 using a multivariate ANOVA. Individual comparison for each subject in all rings was also performed to determine whether there were any significant departures from baseline responses.

The effect of calcium formate ingestion on each electrophysiology test was evaluated by determining means and confidence intervals and comparing each time point of testing with the baseline values.

\section{Results}

Twelve adult females participated in the study. Their mean age was 41 (21-60 years), and mean BMI was $24.3 \pm$ 2.46. They were free of systemic disorders that could potentially affect calcium absorption or underlying serum calcium, such as hyperparathyroidism, bone disease, and intestinal malabsorption. No subject had conditions that could be exacerbated by calcium intake, such as kidney stones. No subject was pregnant. At no time during the study or the week preceding it did any subject ingest vitamin or mineral supplements or other medications that could affect calcium or vitamin D metabolism.

Subjects were fully compliant with the regimen of ingesting 1,300 mg of calcium formate three times a day for 14 consecutive days. There were no serious adverse events noted by history, examination, laboratory tests, or tests of visual function. On two occasions, one subject noted mild bloating 
and abdominal cramping 30 min after ingestion of the calcium supplement. The episodes resolved quickly with eating and did not lead to discontinuation of the supplement. One subject noted two brief episodes of increased perspiration. Another complained of minor headache on two occasions, unrelated temporally with supplement ingestion.

Laboratory evaluations performed to assess subjects for underlying abnormality prior to enrollment were repeated during the study. Assessment of liver, kidney, pancreas, thyroid, parathyroid, and electrolyte status found one subject with mild hypothyroid status at baseline and no subsequent change. All other tests remained essentially unchanged from baseline in all other subjects.

Serum calcium remained stable through the study. At baseline, the mean serum calcium was $9.46 \pm 0.27 \mathrm{mg} / \mathrm{dL}$ (9.0-9.9). At day 15, the mean serum calcium was $9.46 \pm 0.31$ $\mathrm{mg} / \mathrm{dL}$ (8.9-9.9), demonstrating no significant change, $P=0.92$; ionized calcium remained similarly stable (data not shown). This is consistent with numerous studies indicating that oral calcium supplements rarely produce observable alterations in serum calcium levels due to the rapid ability of the body to sequester exogenous calcium entering the bloodstream, thus maintaining serum calcium within normal levels. ${ }^{9}$

Serum formate concentration was evaluated repeatedly to determine if toxic levels were reached and whether accumulation occurred with daily ingestion (Fig. 1). At screening, the mean basal serum formate level was $0.539 \pm 0.06$ $\mathrm{mM}$ (95\% CI 0.497-0.580). Baseline serum formate, prior to ingestion of supplements, demonstrated a slightly higher mean concentration of $0.572 \pm 0.06 \mathrm{mM}$ (95\% CI 0.532-0.613). On the morning of day 15, the mean serum formate level was $0.582 \pm 0.091 \mathrm{mM}$ (95\% CI 0.521-0.642), with no significant difference between this value and the screening level

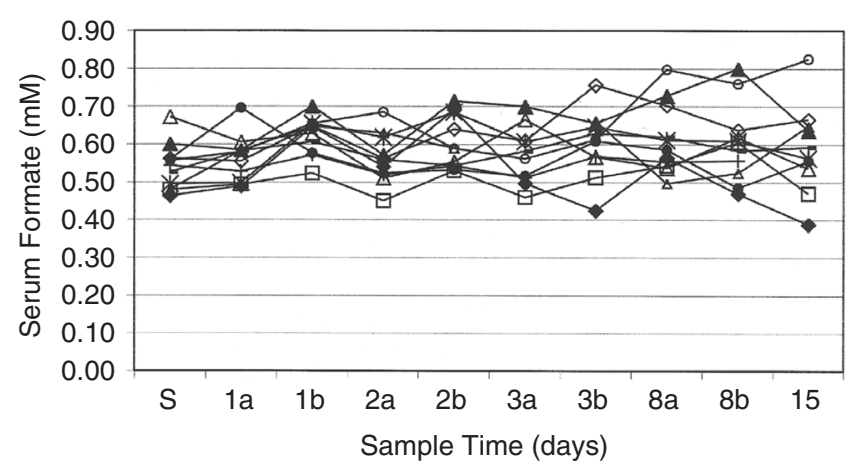

FIG. 1. Serum formate concentrations determined prior to and throughout the 14-day study. The concentrations observed at screening (S) do not vary significantly, either for time groups or for individual subjects, over the course of the study. Observations 1a, 2a, 3a, 8a, and 15 were made prior to the first dose of the day indicated, while observations $1 b, 2 b$, $3 \mathrm{~b}$, and $8 \mathrm{~b}$ were made $40-60 \mathrm{~min}$ after ingesting the second $1,300 \mathrm{mg}$ dose of calcium formate on the day indicated. The sample times "a" and " $b$ " correspond, respectively, to the times of trough and peak levels of serum formate following single doses (see Ref. 36). The data show no accumulation of serum formate with repeated administration of $1,300 \mathrm{mg}$ calcium formate three times per day over 14 days.
$(P=0.268)$. When comparing mean peak serum formate at different time points, a significant difference is found on day 1 with a mean concentration of $0.625 \mathrm{mM}(P=0.01)$ and day 3 with a mean concentration of $0.625 \mathrm{mM}(P=0.03)$ compared with the screening value. These values are not significantly different from the baseline value (day 1, predosing). Comparing trough and peak values for each testing day, there is significant elevation on day $1(P=0.03)$ and day 2 $(P=0.04)$, but not for day $3(P=0.19)$ and day $8(P=0.96)$. However, these differences in serum formate are very small and clinically insignificant. Overall, the results demonstrate that despite the administration of $1,300 \mathrm{mg}$ of calcium formate three times per day, the concentration of serum formate remained clinically stable, with no accumulation over time and no significant elevation beyond the basal levels obtained prior to ingestion.

The physical examination remained unchanged in all subjects, including neurological vitals and pupillary response to light. Blood pressure demonstrated a slight decrease but this was not statistically significant, with baseline blood pressure of $117 \pm 15.1$ systolic and $75.3 \pm 9.7$ diastolic versus a mean of $111 \pm 14.5$ systolic $(P=0.06)$ and $71.7 \pm 9.0$ diastolic at day $15(P=0.12)$.

The ocular examination was normal in all subjects with the exception of one with small macular drusen. All subjects had normal visual acuity and visual function testing at baseline, with no deterioration noted as a group or in any individual when comparing their results to baseline. Masked assessment of fundus photographs found no changes in retinal or optic nerve appearance. The only notable finding when comparing the baseline and final visits was a slight improvement in ETDRS visual acuity from $87.92 \pm 5.9$ to $90.5 \pm 6.5$ letters in the right eye and from $89.0 \pm 4.0$ to $91.4 \pm 4.0$ letters in the left eye $(P=0.007)$. Color vision testing with the FM-100 Hue test found an average of 27.3 errors (22.6 SD, $P=0.027$ ) on day 15 , compared to the initial result of 37.8 errors (17.4 SD) at screening. These color vision results are within the normal range at both time points.

Functional assessment of the optic nerve included Humphrey 24-2 visual fields and VEP. The mean defect at baseline was -1.54 (SD 1.8) for the right eye and -1.36 (SD 1.8) for the left eye, with follow-up results of -1.3 (SD 1.7) for the right eye and -1.34 (SD 1.4) for the left eye $(P=0.33)$. The pattern standard deviation remained similarly unchanged. The latency and amplitude of the VEP were normal for all subjects at baseline and showed no significant changes for all subsequent testing points. At screening the combined latency for both eyes was $175.3 \pm 14.1 \mathrm{~ms}$ versus $174.5 \pm 13.9 \mathrm{~ms}$ on day 2 at peak serum level $(P=0.7)$, and $183.6 \pm 21.0 \mathrm{~ms}$ on day $15(P=0.1)$. The combined amplitude for both eyes was $17.4 \mu \mathrm{V}(95 \% \mathrm{CI} 13.1-21.7)$ at screening versus $16.2 \mu \mathrm{V}(95 \% \mathrm{CI}$ 12.7-19.8) on day $2(P=0.5)$, and $18.8 \mu \mathrm{V}(95 \% \mathrm{CI} 14.5-23.2)$ on day $15(P=0.2)$.

Functional assessment of the retina included full-field and multifocal ERG, as well as pattern ERG to evaluate ganglion function. PERG amplitude for both eyes combined measured $7.7 \pm 6.19 \mu \mathrm{V}$ at baseline versus $5.34 \pm 2.44 \mu \mathrm{V}(P=0.43)$ on final assessment, while latency measured $77.2 \pm 12.3 \mathrm{~ms}$ at baseline versus $69.3 \pm 16.7 \mathrm{~ms}(P=0.21)$ on day 15 . Despite the apparent decrease in mean amplitude and contradictory improvement in latency, the results are not statistically different due to broad standard deviation. Full-field ERG testing (Fig. 2) found no evidence of significant change in retinal 


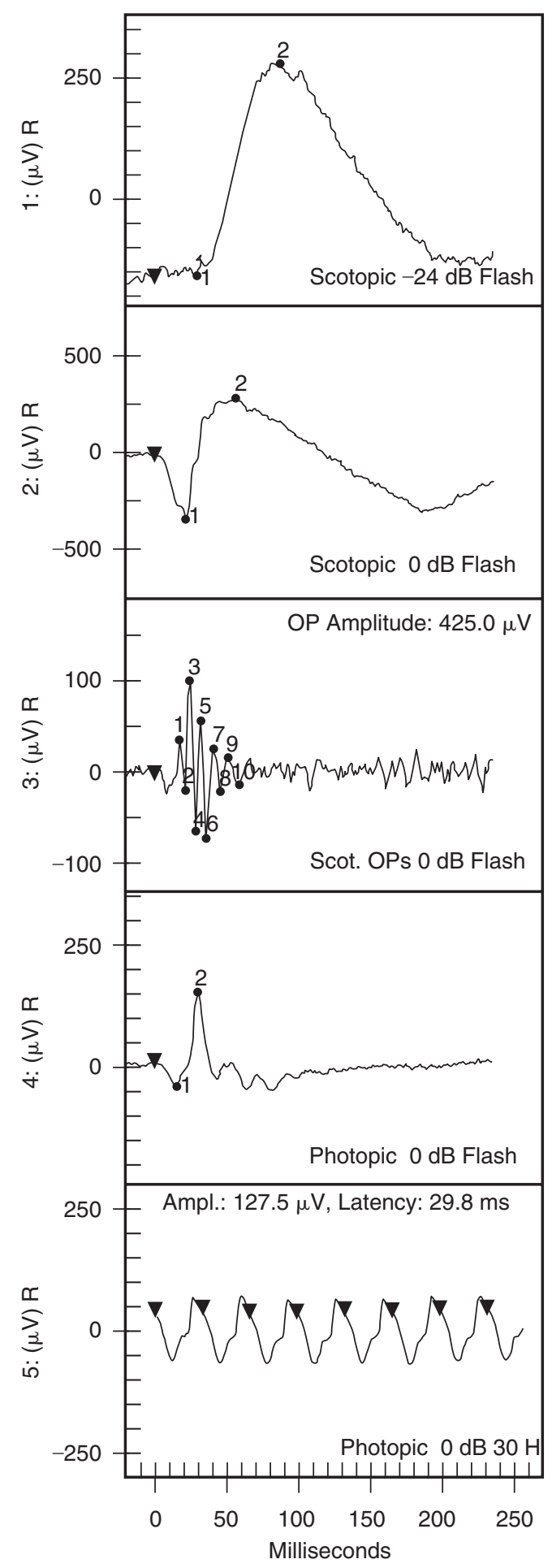

FIG. 2. Normal full-field electroretinogram results in the right eye of subject 1 at Day 8, 45 min after ingestion of the second $1,300 \mathrm{mg}$ dose of calcium formate on that day. This sampling time corresponds to the expected peak level of formate absorption as noted in Figure 1.

function during the study. These results are supported by the stability in the MERG (Fig. 3). Results were analyzed by concentric rings emanating from the fovea, comparing group results between baseline and two follow-up points, as well as comparing individual subject results. Multivariate
ANOVA analysis showed that there were no significant differences in amplitude or latency in the six rings in the postdosing tests compared to baseline.

In summary, formate did not accumulate in the serum with daily dosing, and did not rise significantly above basal levels. Physical and ocular examination, laboratory investigation, and electrophysiological assessments all found that all subjects remained stable throughout the study period.

\section{Discussion}

It is widely recommended that individuals at risk for osteoporosis should ingest 1,200-1,500 mg of elemental calcium daily in their diet. ${ }^{3,18,19}$ However, many diets are deficient in calcium, and absorption of calcium decreases with age, making supplementation necessary. ${ }^{20}$ The salt form of calcium can influence the rate of calcium absorption. ${ }^{21}$ Calcium formate, $\mathrm{Ca}\left(\mathrm{HCO}_{2}\right)_{2}$, is a new water-soluble calcium salt with potential for use as a dietary calcium supplement. ${ }^{8}$ One 650 mg tablet of calcium formate contains $200 \mathrm{mg}$ of elemental calcium and $450 \mathrm{mg}$ formate. Calcium formate has a superior bioavailability profile compared with other common calcium supplements. Being much more soluble than calcium carbonate and much more dissociated in solution than calcium citrate, calcium formate demonstrates superior ability to deliver calcium to the bloodstream after oral administration and may therefore offer significant advantages as a dietary calcium supplement. ${ }^{9}$

The current study was designed to evaluate the safety of oral calcium formate as a calcium supplement, and to determine if there is any accumulation of serum formate with repeated dosing three times per day over a period of 2 weeks. As part of the safety assessment, particular emphasis was placed on detecting possible untoward effects on the visual system, as the retina and optic nerve are the tissues most susceptible to adverse effects of sustained high serum formate concentrations. ${ }^{11,13,22}$

Formate is a normal endogenous metabolite in the body. It distributes readily throughout total body water and under normal conditions appears in plasma or serum at concentrations of up to $0.50 \mathrm{mM}$, reflecting a balance between the production of formate by the catabolism of amino acids and other dietary components and the oxidation of formate to carbon dioxide by all tissues. Exogenously administered formate is primarily oxidized by the liver to $\mathrm{CO}_{2}$ and exhaled; urinary excretion of formate accounts for only $2 \%-4 \%$ of the dose..$^{23}$

Although formate is endogenous to the body, it is toxic if very high serum concentrations are achieved and sustained. In methanol (wood alcohol) poisoning, formic acid is produced and ionizes to release hydrogen ions and formate ions. Methanol poisoning is characterized by profound systemic acidosis, which does not occur with calcium formate ingestion as there are no hydrogen ions to release. In methanolintoxicated subjects, ocular and systemic toxicity can occur following prolonged and substantial elevation of serum formate levels. ${ }^{24}$ Retinal and vitreous humor formate concentrations closely parallel blood formate concentrations. ${ }^{25}$ Studies in vitro and in methanol-intoxicated rodents have shown that formate ion inhibits cytochrome oxidase, the terminal electron acceptor of the mitochondrial electron transport chain essential for mitochondrial energy production. ${ }^{14,26-28}$ This results in depletion of retinal and optic nerve ATP that 
A

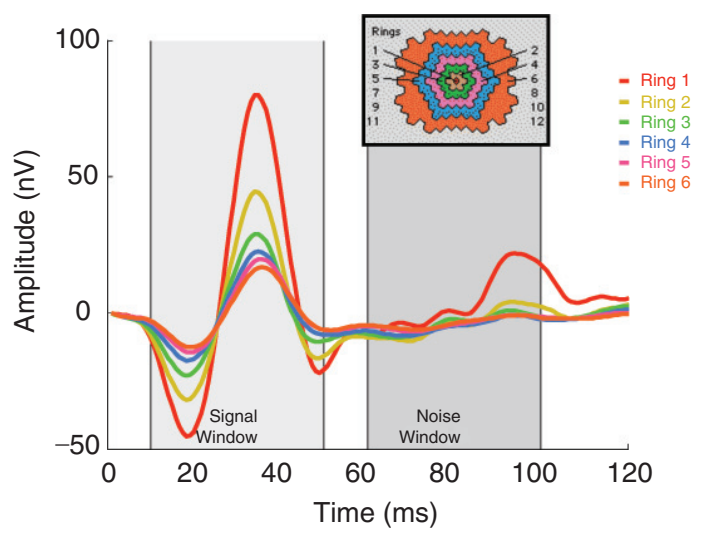

B

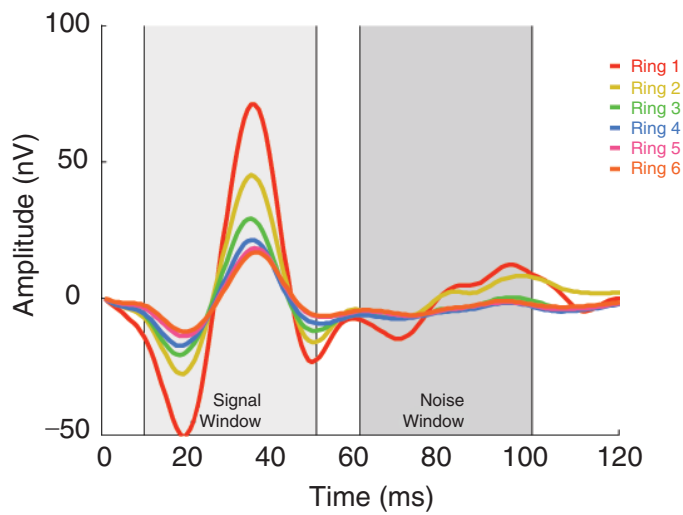

C

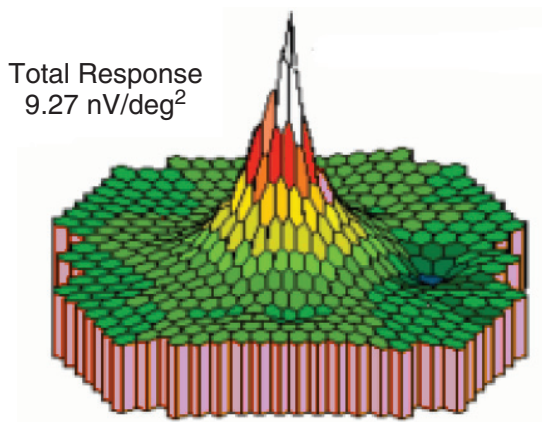

FIG. 3. Average multifocal electroretinogram (MERG) waveforms at baseline (A) and at 15 days (B) for six concentric rings for the right eye. There was no significant difference for either eye at any test point in any ring. The inset shows the spatial arrangement of the ring averages. Ring 1 (red) is the element centered on the fovea, and Ring 6 is the average of the outermost responses (orange). (C) A representative subject on Day 8 with serum formate at peak level demonstrates a normal MERG response.

reduces the activity of the membrane $\mathrm{Na}-\mathrm{K}$ ATPase pump, leading to loss of conduction of the action potential, damage to the myelin sheath, and loss of vision. It also leads to stasis of axoplasmic flow that results in intra-axonal swelling and optic disc edema. ${ }^{29}$ Histological studies have confirmed predominant mitochondrial damage. ${ }^{30,31}$ Optic nerve and retinal damage are identified by loss of pupillary response, optic nerve edema, retinal edema, and hemorrhages, and are corroborated by abnormalities in functional tests including visual acuity, color vision, and visual field abnormalities. ${ }^{11}$ In animal models and case reports of human toxicity, reductions in electroretinogram and VEP responses occurred with severe and extended blood formate accumulation.13,14 Therefore, this study included multiple methods to evaluate both optic nerve and retinal function as calcium formate was administered over 2 weeks.

Formate is a weak inhibitor of cytochrome oxidase with an apparent inhibition constant $\left(K_{\mathrm{i}}\right)$ between 5 and $30 \mathrm{mM} .{ }^{12}$ To have potential for toxicity, serum formate levels must be sustained above $7.0 \mathrm{mM}$ for 24 h. $\cdot^{11,22,32,33}$ Cases of methanol poisoning can achieve serum formate concentrations of $10-20 \mathrm{mM}$ or more. ${ }^{34}$ In contrast, peak serum formate levels after oral administration of a very large single oral dose of calcium formate $(4,550 \mathrm{mg})$ in human subjects averaged $0.7 \pm 0.07 \mathrm{mM} .{ }^{35}$ A pharmacokinetic study of a single large $(3,900 \mathrm{mg})$ oral dose of calcium formate administered to
14 healthy women found that the plasma formate concentration rose quickly but modestly within $30-60 \mathrm{~min}$ and returning to baseline within $<4 \mathrm{~h}$. Thus, the maximum serum formate levels following a single large dose of calcium formate were far below toxic levels and were elevated only transiently, suggesting no liability for accumulation of serum formate from dose to dose. ${ }^{36}$ No adverse effects were noted by any of the subjects in the prior studies.

A major purpose of the present study was to evaluate whether the amount of formate absorbed from repeat dosing of calcium formate presented a toxic challenge to the eye and other organs. The numerous ocular tests were timed to correspond with the peak serum levels of formate. Extensive laboratory testing followed other metabolic parameters that would indicate abnormal underlying function of major organs or an abnormal response to calcium formate ingestion. There were no changes from baseline status in the subjects' endocrine, renal, hepatic, respiratory, and hematologic tests. Serum calcium, ionized calcium, and parathyroid hormone measured prior to the first dose of the day remained stable. Serum formate analyses were performed at the screening examination, at baseline, and at trough and peak periods for serum formate throughout the 2-week study period. There is a basal level of formate in the serum, and a known toxic range. In this study, despite the ingestion of large doses of calcium formate, at no time did the serum 
formate concentration approach the levels required to produce ocular toxicity. The highest single peak serum concentration observed was $0.83 \mathrm{mM}$, and such peaks are transient due to the rapid metabolism of formate to carbon dioxide. In contrast, based on results from cases of methanol poisoning, toxicity to the visual system requires prolonged elevation above $7 \mathrm{mM}$. The peak serum levels on day 1 and day 3 were higher than the screening serum formate, but were equivalent with the mean baseline serum formate level, obtained prior to ingestion of supplement on day 1 . The peak serum level on day 8 and the final level on day 15 were not significantly different than screening or baseline levels, demonstrating a lack of accumulation of formate in the serum.

Formate toxicity, in the setting of methanol poisoning, has been associated with optic nerve abnormality and dysfunction, described as edematous or hyperemic optic nerves, loss of pupillary reflexes, and extinguished VEP in severe cases. $^{29,37}$ VEP testing can identify signs of toxicity before clinical optic neuropathy is evident. ${ }^{38}$ Over the course of this study, the optic nerve appearance remained normal as assessed by the examiner and masked graders. Functional assessment of pupillary response to light as well as objective assessment with VEPs, conducted at times to coincide with peak serum formate concentration, remained normal. Visual fields remained normal, with no significant change in mean defect. Similarly, ganglion cell function, evaluated with pattern ERG, remained unchanged throughout the study. There was no evidence of a deleterious effect of calcium formate on optic nerve anatomy or function.

Dilated retinal examinations were conducted throughout the study and documentary photographs were obtained. Retinal edema was described in many cases in a series of 323 individuals who ingested methanol-contaminated whiskey. Other ocular signs included constricted visual fields, fixed and dilated pupils, and optic nerve edema. Many of these individuals lost their vision. ${ }^{39,40}$ It is notable that visual changes occurred in those with concurrent systemic acidosis, an event that cannot occur with calcium formate ingestion, even at high doses. The current study identified no changes from the normal baseline retinal appearance with repeated calcium formate ingestion. Functional assessment included best-corrected ETDRS visual acuity and color vision testing with the FM-100 Hue test. Interestingly, visual acuity improved in both eyes over the course of the study with statistical significance from $87.92 \pm 5.9$ to $90.5 \pm 6.5$ letters in the right eye and from $89.0 \pm 4.0$ to $91.4 \pm 4.0$ letters in the left eye $(P=0.007)$; this is probably due to a learning effect. Similarly, a slight reduction in the error scores for color vision testing would be accounted for by a learning effect.

Our objective testing of retinal function included fullfield ERG and multifocal ERG to evaluate photoreceptor, bipolar, and Muller cell function. Formate toxicity has been associated with a dose-related reduction in the ERG a-wave (photoreceptor function) and b-wave (bipolar/Muller cell function). ${ }^{14,41,42}$ Both rod-dominated and cone-mediated electroretinogram responses are profoundly attenuated in methanol-intoxicated rats. Histopathologic changes were apparent in photoreceptors and RPE cells, with evidence of inner segment swelling and mitochondrial disruption. ${ }^{30,40,43,44}$ This study found no significant changes in the ERG over time. MERG results corroborated this, indicating that even the isolation of retinal responses into regions did not identify any toxicity. Therefore, both the subjective and objective evaluation of retinal anatomy and retinal function found no evidence of toxicity.

There were several instances of nonserious adverse events attributed to the study compound. Two individuals developed mild transient abdominal cramps that resolved spontaneously, one developed a headache on one occasion, and another had episodes of diaphoresis. The side effects described are common with ingestion of calcium supplements in any form. ${ }^{45}$ The most common side effect of calcium ingestion is constipation, which did not occur in this study. ${ }^{46}$

This preliminary study demonstrates that calcium formate can be ingested repeatedly in moderately high doses with no severe adverse events occurring. Ocular and systemic function as evaluated by numerous parameters remained unchanged. Serum formate levels remained near basal levels, with no evidence of formate accumulation after 2 weeks of repeated use three times per day. This finding is in significant contrast to the accumulation of serum formate that characteristically occurs in methanol poisoning. This study also demonstrates that exogenous formate is well-tolerated when used to promote calcium absorption. Calcium formate is highly bioavailable and can therefore be administered in lower doses. As it is well-tolerated, compliance with a calcium supplementation regimen may be improved. In view of the critical need to increase the rate of calcium supplementation in the population at risk for osteoporosis, ${ }^{47}$ it is important to improve the individual's ability to be compliant with their regimen. This may decrease the morbidity associated with osteopenia and osteoporosis. Prior studies with calcium formate supplementation have demonstrated significantly greater increases in serum calcium concentration and greater bioavailability than equimolar amounts of calcium from supplements based on calcium carbonate or calcium citrate. In combination with the lack of formate accumulation and the lack of toxicity demonstrated in this study, calcium formate may have considerable promise for use as a new dietary calcium supplement.

\section{Conclusion}

Calcium formate is a novel, highly bioavailable calcium salt with potential for use as a dietary calcium supplement. Twelve healthy women participated in an ocular and systemic safety study, ingesting 1,300 $\mathrm{mg}$ of calcium formate three times a day for 2 weeks. The dietary supplement was well-tolerated and safe, with no adverse physical, laboratory, or electrophysiological findings. Evaluation of retinal and optic nerve function included extensive objective testing. The results remained normal throughout the study, with no significant change from baseline. Except for mild transient increases immediately after dosing, serum formate did not accumulate and remained near basal levels. To our knowledge, this is the only study of the effects of administering formate to human subjects in high doses, repeatedly, over 14 days. Since there was no evidence of toxicity in this study, and since calcium formate is highly bioavailable, it may prove to be a good choice for calcium supplementation for maintaining bone integrity. While the absence of serious adverse effects in this study is encouraging, longer term studies with larger groups of subjects representing more varied age, health, dietary, and nutritional status are needed 
to establish the safety of calcium formate for the general population.

\section{Acknowledgments}

University of Wisconsin Investigators: Justin Gottlieb, MD, Barbara Blodi, MD, Thomas Stevens, MD, Suresh Chandra, MD, Michael Ip, MD.

Coordinators: Erika Soderling, Jennie Perry-Raymond. Safety Board: Herbert Chen, MD, Endocrine Surgery, Chair; Michael Nork, MD, MS, Ophthalmology; Don Wiebe, $\mathrm{PhD}$, Toxicology; Bruce Lindsay, MD, Emergency Medicine.

The study was funded by Nephro-Tech 1, LLC, Shawnee, Kansas, which also provided the calcium formate tablets.

\section{Author Disclosure Statement}

Robert Hanzlik is a consultant to Nephro-Tech 1. Other authors have no financial interest in the material presented. The Wisconsin authors had full access to and take full responsibility for the integrity of the data and the accuracy of the data analysis.

\section{References}

1. [No authors listed]. Dietary supplement fact sheet: Calcium. 2005. NIH office of dietary supplements. NIH clinical center. http://ods.Od.Nih.Gov/factsheets/calcium.Asp. Accessed November 16, 2008.

2. Raisz, L. Pathogenesis of osteoporosis: Concepts, conflicts, and prospects. J. Clin. Invest. 115:3318-3325, 2005.

3. [No authors listed]. NIH consensus conference. Optimal calcium intake. J. Am. Med. Assoc. 272:1942-1948, 1994.

4. Jackson, R.D., LaCroix, A.Z., Gass, M., et al. Calcium plus vitamin D supplementation and the risk of fractures. N. Engl. J. Med. 354:669-683, 2006.

5. Prince, R.L., Devine, A., and Dhaliwal, S.S. Effects of calcium supplementation on clinical fractures and bone structure: Results of a 5-year, double-blind, placebo-controlled trial in elderly women. Arch. Int. Med. 166:869-875, 2006.

6. Prince, R.L., Smith, M., Dick, I.M., et al. Prevention of postmenopausal osteoporosis. A comparative study of exercise, calcium supplementation, and hormone replacement therapy. N. Engl. J. Med. 325:1189-1195, 1991.

7. Shea, B., Wells, G., Cranney, A., et al. Meta-analyses of therapies for postmenopausal osteoporosis. Endocrin. Rev. 23:552-559, 2002.

8. DeLuca, H.F. U.S. Patent US 6,528,542 Calcium formate for use as a dietary supplement. March 4, 2003.

9. Hanzlik, R.P., Fowler, S.C., and Fisher, D.H. Relative bioavailability of calcium from calcium formate, calcium citrate, and calcium carbonate. J. Pharmacol. Exp. Therap. 313:1217-1222, 2005.

10. Malorny, G. Stoffwechselversuche mit natrium-formiat und ameisensaure beim menschen. Zeitschrift fur Ernahrungswissenschaft. 9:340-348, 1969.

11. Martin-Amat, G., McMartin, K.E., Hayreh, S.S., et al. Methanol poisoning: Ocular toxicity produced by formate. Toxicol. Appl. Pharmacol. 45:201-208, 1978.

12. Nicholls, P. Formate as an inhibitor of cytochrome $c$ oxidase. Biochem. Biophys. Res. Commun. 67:610-616, 1975.

13. Treichel, J., Murray, T., Lewandowski, M.F., et al. Retinal toxicity in methanol poisoning. Retina. 28:385-385, 2004.

14. Eells, J.T., Henry, M., Lewandowski, M.F., et al. Development and characterization of a rodent model of methanol-induced retinal and optic nerve toxicity. NeuroToxicol. 21:321-330, 2000.

15. Makar, A.B., and Tephly, T.R. Improved estimation of formate in body fluids and tissues. Clin. Chem. 28:385-385, 1982.
16. Kondo, M., Miyake, Y., Horiguchi, M., et al. Clinical evaluation of multifocal electroretinogram. Invest. Ophthalmol. Visual Sci. 36:2146-2150, 1995.

17. Kretschmann, U., Seeliger, M., Ruether, K., et al. Spatial cone activity distribution in diseases of the posterior pole determined by multifocal electroretinography. Vision Res. 38:3817-3828, 1998.

18. [No authors listed]. Bone health and osteoporosis: A report of the surgeongeneral,officeofthesurgeongeneral.2004. Rockville,MD. http://www.Surgeongeneral.Gov/library/bonehealth/content. html. Accessed October 07, 2007.

19. Porter, C. Vitamin and mineral supplements: Part 1. The $R x$ Consultant. 12:1-8, 2003.

20. Heaney, R., Recker, R., Stegman, M., et al. Calcium absorption in women: Relationships to calcium intake, estrogen status, and age. J. Bone Miner. Res. 4:469-475, 1989.

21. Heller, H.J., Stewart, A., Haynes, S., et al. Pharmacokinetics of calcium absorption from two commercial calcium supplements. J. Clin. Pharmacol. 39:1151-1154, 1999.

22. Eells, J.T., Salzman, M.M., Lewandowski, M.F., et al. Formateinduced alterations in retinal function in methanol-intoxicated rats. Toxicol. Appl. Pharmacol. 140:58-69, 1996.

23. Baumann, K., and Angerer, J. Occupational chronic exposure to organic solvents. Vi. Formic acid concentration in blood and urine as an indicator of methanol exposure. Int. Arch. Occup. Environ. Health. 42:241-249, 1979.

24. Barceloux, D.G., Bond, G.R., Krenzelok, E.P., et al. American academy of clinical toxicology practice guidelines on the treatment of methanol poisoning. Clin. Toxicol. 40:415-446, 2002.

25. Seme, M.T., Summerfelt, P., Neitz, J., et al. Differential recovery of retinal function after mitochondrial inhibition by methanol intoxication. Invest. Ophthalmol. Visual Sci. 42:834-841, 2001.

26. Johlin, F.C., Fortman, C.S., Nghiem, D.D., et al. Studies on the role of folic acid and folate-dependent enzymes in human methanol poisoning. Mol. Pharmacol. 31:557-561, 1987.

27. Liesivouri, J., and Savolainen, H. Methanol and formic acid toxicity: Biochemical mechanisms. Pharm. Toxicol. 69:157-163, 1991.

28. Tephly, T.R. The toxicity of methanol. Life Sci. 48:1031-1041, 1991.

29. Hayreh, M.S., Hayreh, S.S., Baumbach, G.L., et al. Methyl alcohol poisoning iii. Ocular toxicity. Arch. Ophthalmol. 95:1851-1858, 1977.

30. Sharpe, J.A., Hstovsky, M., Bilbao, J.M., et al. Methanol optic neuropathy: A histopathological study. Neurology. 32:1093-1100, 1982.

31. Treichel, J.L., Henry, M.M., Skumatz, C.M.B., et al. Formate, the toxic metabolite of methanol, in cultured ocular cells. NeuroToxicol. 24:825-834, 2003.

32. Jacobson, D., and McMartin, K.E. Methanol and ethylene glycol poisonings: Mechanism of toxicity, clinical course, diagnosis and treatment. Med. Toxicol. 1:309-344, 1986.

33. Kavat, R., and Nauss, K. The toxicity of methanol vapors. CRC Crit. Rev. Toxicol. 21:21-50, 1990.

34. Sejersted, O.M., Jacobsen, D., Ovrebo, S., et al. Formate concentrations in plasma from patients poisoned with methanol. Acta Med. Scand. 213:105-110, 1983.

35. [No authors listed]. Data on file, Nephro-Tech 1, LLC, 2003.

36. Hanzlik, R.P., Fowler, R.P., and Eells, J.T. Absorption and elimination of formate following oral administration of calcium formate in female human subjects. Drug Metab. Dispos. 33:282-286, 2005.

37. Hantson, P., de Tourtchaninoff, M., Simoens, G., et al. Evoked potentials investigation of visual dysfunction after methanol poisoning. Crit. Care Med. 27:2707-2715, 1999.

38. McKellar, M.J., Hidajat, R.R., and Elder, M.J. Acute ocular methanol toxicity: Clinical and electrophysiological features. Aust. N.Z. J. Ophthalmol. 25:225-230, 1997.

39. Bennett, Jr., J.L., Cary, F.H., Mitchell, G.L., et al. Acute methyl alcohol poisoning: A review based on experiences in an outbreak of 323 cases. Medicine. 32:431-463, 1953. 
40. Benton, Jr., C.D., and Calhoun, Jr., F.P. The ocular effects of methyl alcohol poisoning. Report of a catastrophe involving 320 persons. Am. J. Ophthalmol. 36:1677-1685, 1952.

41. Ruedemann, A.D. The electroretinogram in chronic methyl alcohol poisoning in human beings. Trans. Am. Ophthalmol. Soc. 59:480-529, 1961.

42. Seme, M.A., Summerfelt, P., Henry, M.M., et al. Formate-induced inhibition of photoreceptor function in methanol intoxication. J. Pharmacol. Exp. Therap. 289:361-370, 1999.

43. Gardner, C.D., Lee, E.W., and Louis-Ferdinand, R.T. Muller cell involvement in methanol induced retinal toxicity. Toxicol. Appl. Pharmacol. 130:101-107, 1995.

44. Murray, T.G., Burton, T.C., Rajani, C., et al. Methanol poisoning: A rodent model with structural evidence for retinal involvement. Arch. Ophthalmol. 109:1012-1016, 1991.

45. Carlon, G.C., Howland, W.S., Goldner, P.L., et al. Adverse effects of calcium administration. Arch. Surg. 113:882-885, 1978.
46. Sheikh, M.S., Santa Ana, C.A., and Nicar, M.J. Gastrointestinal absorption of calcium from milk and calcium salts. N. Engl. J. Med. 316:532-536, 1987.

47. Sunyecz, J.A., and Weisman, S.M. The role of calcium in osteoporosis drug therapy. J. Women's Health. 14:2, 2005.

Received: December 16, 2008

Accepted: March 3, 2009

Reprint Requests: Dr. Robert P. Hanzlik Department of Medicinal Chemistry

University of Kansas

1251 Wescoe Hall Drive

MDCM 4048 Malott

Lawrence, KS 66045

E-mail: rhanzlik@ku.edu 